\title{
Pacific
}

Journal of

Mathematics

\section{A NEW PROBABILITY DISTRIBUTION WITH APPLICATIONS}

MINGJIN WANG

Volume $247 \quad$ No. 1 


\title{
A NEW PROBABILITY DISTRIBUTION WITH APPLICATIONS
}

\author{
MINGJIN WANG
}

\begin{abstract}
We introduce a new probability distribution, which is useful in the study of basic hypergeometric series. As applications, we give probabilistic derivations of the $q$-binomial theorem, the $q$-Gauss summation formula, a new multiple identity, and an extension of the Rogers-Ramanujan identities.
\end{abstract}

\section{Introduction}

The probabilistic method is a useful tool in the study of basic hypergeometric series [Chapman 2005; Evans 2002; Fulman 2001; Rawlings 1997]. In this paper, we introduce a new probability distribution and then demonstrate the applications of this distribution in $q$-series. We begin with recall some definitions, notations and known results in [Andrews et al. 1999; Gasper and Rahman 1990; Liu 2003]. Throughout the paper, we suppose that $0<q<1$. The $q$-shifted factorials are defined as

$$
(a ; q)_{0}=1, \quad(a ; q)_{n}=\prod_{k=0}^{n-1}\left(1-a q^{k}\right), \quad(a ; q)_{\infty}=\prod_{k=0}^{\infty}\left(1-a q^{k}\right) .
$$

We also adopt a compact notation for multiple $q$-shifted factorials:

$$
\left(a_{1}, a_{2}, \ldots, a_{m} ; q\right)_{n}=\left(a_{1} ; q\right)_{n}\left(a_{2} ; q\right)_{n} \cdots\left(a_{m} ; q\right)_{n},
$$

where $n$ is an integer or $\infty$. The $q$-binomial coefficient is defined by

$$
\left[\begin{array}{l}
n \\
k
\end{array}\right]=\frac{(q ; q)_{n}}{(q ; q)_{k}(q ; q)_{n-k}}
$$

In 1846, Heine introduced the ${ }_{r+1} \phi_{r}$ basic hypergeometric series, which is defined by

$$
{ }_{r+1} \phi_{r}\left(\begin{array}{c}
a_{1}, a_{2}, \ldots, a_{r+1} \\
b_{1}, b_{2}, \ldots, b_{r}
\end{array} ; q, x\right)=\sum_{n=0}^{\infty} \frac{\left(a_{1}, a_{2}, \ldots, a_{r+1} ; q\right)_{n} x^{n}}{\left(q, b_{1}, b_{2}, \ldots, b_{r} ; q\right)_{n}}
$$

MSC2000: primary 60E05; secondary 33D15, 05A10.

Keywords: probability distribution, basic hypergeometric series, Andrews-Askey integral,

Al-Salam-Carlitz polynomials, Lebesgue's dominated convergence theorem, Tannery's theorem, Rogers-Ramanujan identities. 
F. H. Jackson [1910] defined the $q$-integral by

$$
\int_{0}^{d} f(t) d_{q} t=d(1-q) \sum_{n=0}^{\infty} f\left(d q^{n}\right) q^{n}
$$

and

$$
\int_{c}^{d} f(t) d_{q} t=\int_{0}^{d} f(t) d_{q} t-\int_{0}^{c} f(t) d_{q} t .
$$

The $q$-integrals are important in the theory and application of basic hypergeometric series. For example, the author gives some applications of the $q$-integral in [Wang 2008; 2009b; 2009a; 2010b; 2010a]. The Andrews-Askey [1981] integral is

$$
\int_{c}^{d} \frac{(q t / c, q t / d ; q)_{\infty}}{(a t, b t ; q)_{\infty}} d_{q} t=\frac{d(1-q)(q, d q / c, c / d, a b c d ; q)_{\infty}}{(a c, a d, b c, b d ; q)_{\infty}}
$$

which can be derived from Ramanujan's ${ }_{1} \psi_{1}$ summation provided that no zero factors occur in the denominator of the integral.

The Al-Salam-Carlitz polynomials $\varphi_{n}^{(a)}(x \mid q)$ are defined by

$$
\varphi_{n}^{(a)}(x \mid q)=\sum_{k=0}^{n}\left[\begin{array}{l}
n \\
k
\end{array}\right] x^{k}(a ; q)_{k},
$$

[Srivastava and Jain 1989] and have the $q$-integral representation [Wang 2009b]

$$
\varphi_{n}^{(a)}(x \mid q)=\frac{(a x, a ; q)_{\infty}}{(1-q)(q, q / x, x ; q)_{\infty}} \int_{x}^{1} \frac{(q t / x, q t ; q)_{\infty} t^{n}}{(a t ; q)_{\infty}} d_{q} t
$$

provided that no zero factors occur in the denominator.

We frequently use the following well-known theorems:

Theorem (analytic continuation theorem). If $f$ and $g$ are analytic at $z_{0}$ and agree at infinitely many points, which include $z_{0}$ as an accumulation point, then $f=g$.

Theorem (Lebesgue's dominated convergence theorem). Suppose that $\left\{X_{n}, n \geq 1\right\}$ is a sequence of random variables such that $X_{n} \rightarrow X$ pointwise almost everywhere as $n \rightarrow \infty$, and such that $\left|X_{n}\right| \leq Y$ for all $n$, where the random variable $Y$ is integrable. Then $X$ is integrable, and

$$
\lim _{n \rightarrow \infty} E X_{n}=E X,
$$

where $E(\cdot)$ denotes expected value.

Tannery's theorem is a special case of Lebesgue's dominated convergence theorem on the sequence space $L^{1}$. 
Theorem [Tannery 1904]. If $s(n)=\sum_{k \geq 0} f_{k}(n)$ is a finite sum (or a convergent series) for each $n$,

$$
\lim _{n \rightarrow \infty} f_{k}(n)=f_{k}, \quad\left|f_{k}(n)\right| \leq M_{k}, \quad \text { and } \quad \sum_{k=0}^{\infty} M_{k}<\infty
$$

then

$$
\lim _{n \rightarrow \infty} s(n)=\sum_{k=0}^{\infty} f_{k}
$$

\section{A new probability distribution}

In order to use Lebesgue's dominated convergence theorem to get $q$-identities, we need to find some special probability distributions. In this section, we introduce a useful probability distribution.

The main method of this paper as follows: First, we define a probability distribution by $q$-shifted factorials; its expected value can be easily obtained. Then we construct a sequence of random variables with this probability distribution. Finally, we use Lebesgue's dominated convergence theorem to obtain a $q$-identity.

Lemma 2.1. Suppose $x$ is a real such that $x<0$; then we have

$$
\frac{(-x)^{n}\left(x^{n-1} q^{k+1}, x^{n} q^{k+1} ; q\right)_{\infty} q^{k}}{(q, q / x, x ; q)_{\infty}} \geq 0
$$

and

$$
\sum_{n=0}^{1} \sum_{k=0}^{\infty} \frac{(-x)^{n}\left(x^{n-1} q^{k+1}, x^{n} q^{k+1} ; q\right)_{\infty} q^{k}}{(q, q / x, x ; q)_{\infty}}=1,
$$

where $n=0,1$ and $k=0,1,2, \ldots$

Proof. Inequality (2-1) is obvious by the definition of the $q$-shifted factorials and the assumption that $x<0$. We only need to prove (2-2).

Since

$$
\begin{aligned}
& \sum_{n=0}^{1} \sum_{k=0}^{\infty} \frac{(-x)^{n}\left(x^{n-1} q^{k+1}, x^{n} q^{k+1} ; q\right)_{\infty} q^{k}}{(q, q / x, x ; q)_{\infty}} \\
= & \frac{1}{(1-q)(q, q / x, x ; q)_{\infty}} \\
& \times\left((1-q) \sum_{k=0}^{\infty}\left(q^{k+1} / x, q^{k+1} ; q\right)_{\infty} q^{k}-x(1-q) \sum_{k=0}^{\infty}\left(q^{k+1}, x q^{k+1} ; q\right)_{\infty} q^{k}\right),
\end{aligned}
$$


using the definition of the $q$-integral gives

$$
(1-q) \sum_{k=0}^{\infty}\left(q^{k+1} / x, q^{k+1} ; q\right)_{\infty} q^{k}=\int_{0}^{1}(q t / x, q t ; q)_{\infty} d_{q} t
$$

and

$$
x(1-q) \sum_{k=0}^{\infty}\left(q^{k+1}, x q^{k+1} ; q\right)_{\infty} q^{k}=\int_{0}^{x}(q t / x, q t ; q)_{\infty} d_{q} t
$$

Consequently, we have

$$
\begin{aligned}
& (1-q) \sum_{k=0}^{\infty}\left(q^{k+1} / x, q^{k+1} ; q\right)_{\infty} q^{k} \\
& =\int_{0}^{1}(q t / x, q t ; q)_{\infty} d_{q} t-\int_{0}^{x}(q t / x, q t ; q)_{\infty} d_{q} t=\int_{x}^{1}(q t / x, q t ; q)_{\infty} d_{q} t
\end{aligned}
$$

Employing the Andrews-Askey integral (1-3) gives

$$
\int_{x}^{1}(q t / x, q t ; q)_{\infty} d_{q} t=(1-q)(q, q / x, x ; q)_{\infty}
$$

Substituting (2-4) and (2-5) into (2-3) gives (2-2).

Definition 2.2. A random variable $\xi$ has distribution $W(x ; q)$ if

$$
P\left(\xi=x^{n} q^{k}\right)=\frac{(-x)^{n}\left(x^{n-1} q^{k+1}, x^{n} q^{k+1} ; q\right)_{\infty} q^{k}}{(q, q / x, x ; q)_{\infty}},
$$

where $x<0,0<q<1, n=0,1$ and $k=0,1,2, \ldots$

The distribution $W(x ; q)$ has some applications in the study of basic hypergeometric series.

Before giving applications, we need the following lemmas.

Lemma 2.3. Let $-1<x<0$ and $|a|<1$. Let $\xi$ denote a random variable having with $W(x ; q)$. Then we have

$$
E\left(\frac{\xi^{m}}{(a \xi ; q)_{\infty}}\right)=\frac{1}{(a, a x ; q)_{\infty}} \varphi_{m}^{(a)}(x \mid q) \quad \text { for } m=0,1,2, \ldots
$$


Proof. Using the definition of the $q$-integral (1-1), (1-2) and the $q$-integral representation of the Al-Salam-Carlitz polynomials (1-4), we have

$$
\begin{aligned}
& E\left(\frac{\xi^{m}}{(a \xi ; q)_{\infty}}\right) \\
& \quad=\sum_{n=0}^{1} \sum_{k=0}^{\infty} \frac{(-x)^{n}\left(x^{n-1} q^{k+1}, x^{n} q^{k+1} ; q\right)_{\infty} q^{k}}{(q, q / x, x ; q)_{\infty}} \cdot \frac{x^{n m} q^{k m}}{\left(a x^{n} q^{k} ; q\right)_{\infty}} \\
& \quad=\frac{1}{(1-q)(q, q / x, x ; q)_{\infty}}\left((1-q) \sum_{k=0}^{\infty}\left(q^{k+1} / x, q^{k+1} ; q\right)_{\infty} \cdot \frac{q^{k(m+1)}}{\left(a q^{k} ; q\right)_{\infty}}\right. \\
& \left.\quad=\frac{1}{(1-q)(q, q / x, x ; q)_{\infty}} \quad x(1-q) \sum_{k=0}^{\infty}\left(q^{k+1}, x q^{k+1} ; q\right)_{\infty} \cdot \frac{x^{m} q^{k(m+1)}}{\left(a x q^{k} ; q\right)_{\infty}}\right) \\
& \left.\quad=\frac{1}{(1-q)(q, q / x, x ; q)_{\infty}} \int_{x}^{1} \frac{(q t / x, q t ; q)_{\infty} t^{m}}{(a t ; q)_{\infty}} d_{q} t-\int_{0}^{x} \frac{(q t / x, q t ; q)_{\infty} t^{m}}{(a t ; q)_{\infty}} d_{q} t\right) \\
& \quad=\frac{1}{(a, a x ; q)_{\infty}} \varphi_{m}^{(a)}(x \mid q) .
\end{aligned}
$$

Lemma 2.4. Let $-1<x<0$ and $|a|<1$. Let $\xi$ denote a random variable having distribution $W(x ; q)$. Then we have

$$
E\left(\frac{1}{(a \xi, b \xi ; q)_{\infty}}\right)=\frac{(a b x, ; q)_{\infty}}{(a, b, a x, b x ; q)_{\infty}}
$$

Proof. Using the definition of the $q$-integral (1-1), (1-2) and the Andrews-Askey integral (1-3), we have

$$
\begin{aligned}
& E\left(\frac{1}{(a \xi, b \xi ; q)_{\infty}}\right) \\
& =\sum_{n=0}^{1} \sum_{k=0}^{\infty} \frac{(-x)^{n}\left(x^{n-1} q^{k+1}, x^{n} q^{k+1} ; q\right)_{\infty} q^{k}}{(q, q / x, x ; q)_{\infty}} \cdot \frac{1}{\left(a x^{n} q^{k}, b x^{n} q^{k} ; q\right)_{\infty}} \\
& =\frac{1}{(1-q)(q, q / x, x ; q)_{\infty}}\left((1-q) \sum_{k=0}^{\infty}\left(q^{k+1} / x, q^{k+1} ; q\right)_{\infty} \cdot \frac{q^{k}}{\left(a q^{k}, b q^{k} ; q\right)_{\infty}}\right. \\
& \left.-x(1-q) \sum_{k=0}^{\infty}\left(q^{k+1}, x q^{k+1} ; q\right)_{\infty} \cdot \frac{q^{k}}{\left(a x q^{k}, b x^{n} q^{k} ; q\right)_{\infty}}\right)
\end{aligned}
$$




$$
\begin{aligned}
& =\frac{1}{(1-q)(q, q / x, x ; q)_{\infty}}\left(\int_{0}^{1} \frac{(q t / x, q t ; q)_{\infty}}{(a t, b t ; q)_{\infty}} d_{q} t-\int_{0}^{x} \frac{(q t / x, q t ; q)_{\infty}}{(a t, b t ; q)_{\infty}} d_{q} t\right) \\
& =\frac{1}{(1-q)(q, q / x, x ; q)_{\infty}} \int_{x}^{1} \frac{(q t / x, q t ; q)_{\infty}}{(a t, b t ; q)_{\infty}} d_{q} t=\frac{(a b x, ; q)_{\infty}}{(a, b, a x, b x ; q)_{\infty}}
\end{aligned}
$$

which completes the proof.

Lemma 2.5. Let $|x|<1$. Then

$$
\lim _{n \rightarrow \infty} \varphi_{n}^{(a)}(x \mid q)=\sum_{k=0}^{\infty} \frac{(a ; q)_{k} x^{k}}{(q ; q)_{k}} .
$$

Proof. Let $f_{k}(n)=\left[\begin{array}{l}n \\ k\end{array}\right] x^{k}(a ; q)_{k}$ if $k \leq n$ and $f_{k}(n)=0$ if $k>n$. We have

Since

$$
\varphi_{n}^{(a)}(x \mid q)=\sum_{k=0}^{\infty} f_{k}(n) .
$$

$$
\lim _{n \rightarrow \infty} f_{k}(n)=\frac{(a ; q)_{k} x^{k}}{(q ; q)_{k}}, \quad\left|f_{k}(n)\right| \leq \frac{\left|(a ; q)_{k} x^{k}\right|}{(q ; q)_{\infty}}, \quad \sum_{k=0}^{\infty} \frac{\left|(a ; q)_{k} x^{k}\right|}{(q ; q)_{\infty}}<\infty,
$$

by Tannery's theorem we know (2-7) holds.

\section{The $q$-binomial theorem}

One of the most important summation formulas for basic hypergeometric series is the $q$-binomial theorem, which was derived by Cauchy in 1843, Heine in 1847, and by other mathematicians. There are many proofs. By using the probability distribution $W(x ; q)$ and the Lebesgue dominated convergence theorem, we give a probabilistic derivation; see also [Andrews et al. 1999; Gasper and Rahman 1990].

Theorem 3.1. $\sum_{n=0}^{\infty} \frac{(a ; q)_{n} x^{n}}{(q ; q)_{n}}=\frac{(a x ; q)_{\infty}}{(x ; q)_{\infty}}$ for $|x|<1$.

Proof. Let $\xi$ be a random variable having distribution $W(x ; q)$, where $-1<x<0$. We consider the sequence

$$
\left\{\frac{\xi^{n}}{(a \xi ; q)_{\infty}}\right\}_{n=1}^{\infty} \text { for }|a|<1
$$

of random variables (on a probability space). It is easy to see that $\xi^{n}$ converges to $I_{(\xi=1)}$, which has Binomial distribution $B\left(1,1 /(x ; q)_{\infty}\right)$ and

$$
\lim _{n \rightarrow \infty} \frac{\xi^{n}}{(a \xi ; q)_{\infty}}=\frac{I_{(\xi=1)}}{(a ; q)_{\infty}}
$$


where $I_{\Omega}$ is the indicator function defined by

$$
I_{\Omega}(x)= \begin{cases}1 & \text { if } x \in \Omega \\ 0 & \text { if } x \notin \Omega .\end{cases}
$$

Since

$$
\left|\frac{\xi^{n}}{(a \xi ; q)_{\infty}}\right| \leq \frac{1}{(|a| ; q)_{\infty}}
$$

using Lebesgue's dominated convergence theorem gives

$$
\lim _{n \rightarrow \infty} E\left(\frac{\xi^{n}}{(a \xi ; q)_{\infty}}\right)=E\left(\frac{I_{(\xi=1)}}{(a ; q)_{\infty}}\right) .
$$

Employing (1-4) and using Tannery's theorem gives

$$
\begin{aligned}
\lim _{m \rightarrow \infty} E\left(\frac{\xi^{m}}{(a \xi ; q)_{\infty}}\right) & =\frac{1}{(a, a x ; q)_{\infty}} \lim _{m \rightarrow \infty} \varphi_{m}^{(a)}(x \mid q) \\
& =\frac{1}{(a, a x ; q)_{\infty}} \sum_{m=0}^{\infty} \frac{(a ; q)_{m} x^{m}}{(q ; q)_{m}}
\end{aligned}
$$

By direct calculation,

$$
E\left(\frac{I_{(\breve{\xi}=1)}}{(a ; q)_{\infty}}\right)=\frac{1}{(a, x ; q)_{\infty}} .
$$

Substituting (3-2) and (3-3) into (3-1) gives

$$
\sum_{n=0}^{\infty} \frac{(a ; q)_{n} x^{n}}{(q ; q)_{n}}=\frac{(a x ; q)_{\infty}}{(x ; q)_{\infty}},
$$

where $-1<x<0$ and $|a|<1$. By analytic continuation, we may replace the assumptions $-1<x<0$ by $|a|<1$ by $|x|<1$. Thus, we get Theorem 3.1.

\section{The $q$-Gauss summation formula}

In 1847, Heine derived a $q$-analogue of Gauss's summation formula. We show that this result can be recovered with the probability distribution $W(x ; q)$.

Theorem 4.1. ${ }_{2} \phi_{1}\left(\begin{array}{c}a, b \\ c\end{array} ; q, \frac{c}{a b}\right)=\frac{(c / a, c / b ; q)_{\infty}}{(c, c / a b ; q)_{\infty}}$ for $|c /(a b)|<1$.

Proof. Let $\xi$ and $\eta$ denote two independent random variables having distributions $W(x ; q)$ and $W(y ; q)$, respectively, where we set $-1<x, y<0$. We consider the following sequence of random variables (on a probability space):

$$
\left\{\frac{\eta^{n}}{(a \xi \eta ; q)_{\infty}}\right\}_{n=1}^{\infty} \text { for }|a|<1 \text {. }
$$


Clearly $\eta^{n}$ converges to $I_{(\eta=1)}$ having binomial distribution $B\left(1,1 /\left((y ; q)_{\infty}\right)\right)$ and

$$
\lim _{n \rightarrow \infty} \frac{\eta^{n}}{(a \xi \eta ; q)_{\infty}}=\frac{I_{(\eta=1)}}{(a \xi ; q)_{\infty}}
$$

where $I_{\Omega}$ is the indicator function.

Since

$$
\left|\frac{\eta^{n}}{(a \xi \eta ; q)_{\infty}}\right| \leq \frac{1}{(|a| ; q)_{\infty}}
$$

using Lebesgue's dominated convergence theorem gives

$$
\lim _{n \rightarrow \infty} E\left(\frac{\eta^{n}}{(a \xi \eta ; q)_{\infty}}\right)=E\left(\frac{I_{(\eta=1)}}{(a \xi ; q)_{\infty}}\right) .
$$

Observe that

$$
\begin{aligned}
E\left(\frac{\eta^{n}}{(a \xi \eta ; q)_{\infty}}\right) & =E\left(E\left(\frac{\eta^{n}}{(a \xi \eta ; q)_{\infty}} \mid \xi\right)\right) \\
& =E\left(\frac{1}{(a \xi, a y \xi ; q)_{\infty}} \varphi_{n}^{(a \xi)}(x \mid q)\right) \\
& =\sum_{k=0}^{n}\left[\begin{array}{l}
n \\
k
\end{array}\right] y^{k} \cdot E\left(\frac{1}{\left(a \xi q^{k}, a y \xi ; q\right)_{\infty}}\right) \\
& =\sum_{k=0}^{n}\left[\begin{array}{l}
n \\
k
\end{array}\right] y^{k} \cdot \frac{\left(a^{2} x y q^{k} ; q\right)_{\infty}}{\left(a q^{k}, a x q^{k}, a y, a x y ; q\right)_{\infty}} \\
& =\frac{\left(a^{2} x y ; q\right)_{\infty}}{(a, a x, a y, a x y ; q)_{\infty}} \sum_{k=0}^{n}\left[\begin{array}{l}
n \\
k
\end{array}\right] \cdot \frac{(a, a x ; q)_{\infty} y^{k}}{\left(a^{2} x y ; q\right)_{\infty}}
\end{aligned}
$$

Hence, we get the left hand side of (4-1):

$$
\lim _{n \rightarrow \infty} E\left(\frac{\eta^{n}}{(a \xi \eta ; q)_{\infty}}\right)=\frac{\left(a^{2} x y ; q\right)_{\infty}}{(a, a x, a y, a x y ; q)_{\infty}} \sum_{k=0}^{\infty} \frac{(a, a x ; q)_{\infty} y^{k}}{\left(q, a^{2} x y ; q\right)_{\infty}} .
$$

On the other hand, the right hand side of (4-1) equals

$$
E\left(\frac{I_{(\eta=1)}}{(a \xi ; q)_{\infty}}\right)=p(\eta=1) E\left(\frac{1}{(a \xi ; q)_{\infty}}\right)=\frac{1}{(a, a x, y ; q)_{\infty}} .
$$

Substituting (4-2) and (4-3) into (4-1) gives

$$
\sum_{k=0}^{\infty} \frac{(a, a x ; q)_{\infty} y^{k}}{\left(q, a^{2} x y ; q\right)_{\infty}}=\frac{(a y, a x y ; q)_{\infty}}{\left(a^{2} x y, y ; q\right)_{\infty}},
$$

which is equivalent to the $q$-Gauss theorem, Theorem 4.1, by analytic continuation. 


\section{A multiple identity}

Multiple basic hypergeometric series have been investigated by various authors [Milne 1997; Wang 2009a; Zhang 2006; Zhang and Liu 2006]. We will use the distribution $W(x ; q)$ to prove the following multiple identity.

Theorem 5.1. Let $|a|<1$. Then for any positive integers $m$ and $n$, we have

$$
\begin{aligned}
\sum_{y_{1}+\cdots+y_{m} \geq n}\left[\begin{array}{c}
y_{1}+\cdots+y_{m} \\
n
\end{array}\right] q^{y_{2}+2 y_{3}+\cdots+(m-1) y_{m}} a^{y_{1}+\cdots+y_{m}} & \\
& =\frac{a^{n}}{(a ; q)_{n+m}}\left[\begin{array}{c}
n+m-1 \\
n
\end{array}\right] .
\end{aligned}
$$

Proof. Let $\xi$ denote a random variable with distribution $W(x ; q)$, where $-1<x<0$. For any positive integer $m$, we consider the sequence

$$
\left\{\frac{\left(1-(a \xi)^{n}\right)\left(1-(a q \xi)^{n}\right) \cdots\left(1-\left(a q^{m-1} \xi\right)^{n}\right)}{(a \xi ; q)_{\infty}}\right\}_{n=1}^{\infty} \quad \text { for }|a|<1
$$

of random variables (on a probability space). It is easy to see that

$$
\lim _{n \rightarrow \infty} \frac{\left(1-(a \xi)^{n}\right)\left(1-(a q \xi)^{n}\right) \cdots\left(1-\left(a q^{m-1} \xi\right)^{n}\right)}{(a \xi ; q)_{\infty}}=\frac{1}{(a \xi ; q)_{\infty}} .
$$

Since $\left|\left(1-(a \xi)^{n}\right)\left(1-(a q \xi)^{n}\right) \cdots\left(1-\left(a q^{m-1} \xi\right)^{n}\right) /(a \xi ; q)_{\infty}\right| \leq 1 /(|a| ; q)_{\infty}$, using Lebesgue's dominated convergence theorem gives

(5-2) $\lim _{n \rightarrow \infty} E\left(\frac{\left(1-(a \xi)^{n}\right)\left(1-(a q \xi)^{n}\right) \cdots\left(1-\left(a q^{m-1} \xi\right)^{n}\right)}{(a \xi ; q)_{\infty}}\right)=E\left(\frac{1}{(a \xi ; q)_{\infty}}\right)$.

Employing (2-6), we get the right hand side of (5-2):

$$
E\left(\frac{1}{(a \xi ; q)_{\infty}}\right)=\frac{1}{(a, a x ; q)_{\infty}} .
$$

On the other hand, observing that

$$
\begin{aligned}
\frac{\left(1-(a \xi)^{n}\right)\left(1-(a q \xi)^{n}\right) \cdots\left(1-\left(a q^{m-1} \xi\right)^{n}\right)}{(a \xi ; q)_{\infty}} \\
=\frac{1-(a \xi)^{n}}{1-a \xi} \cdot \frac{1-(a q \xi)^{n}}{1-a q \xi} \cdots \frac{1-\left(a q^{m-1} \xi\right)^{n}}{1-a q^{m-1} \xi} \cdot \frac{1}{\left(a q^{m} \xi ; q\right)_{\infty}} \\
=\sum_{y_{1}=0}^{n-1}(a \xi)^{y_{1}} \cdot \sum_{y_{2}=0}^{n-1}(a q \xi)^{y_{2}} \cdots \sum_{y_{m}=0}^{n-1}\left(a q^{m-1} \xi\right)^{y_{m}} \cdot \frac{1}{\left(a q^{m} \xi ; q\right)_{\infty}} \\
=\sum_{0 \leq y_{1}, \ldots, y_{m} \leq n-1} q^{y_{2}+2 y_{3}+\cdots+(m-1) y_{m}} a^{y_{1}+\cdots+y_{m}} \cdot \frac{\xi^{y_{1}+\cdots+y_{m}}}{\left(a q^{m} \xi ; q\right)_{\infty}},
\end{aligned}
$$


we have

$$
\begin{gathered}
E\left(\frac{\left[1-(a \xi)^{n}\right]\left[1-(a q \xi)^{n}\right] \cdots\left[1-\left(a q^{m-1} \xi\right)^{n}\right]}{(a \xi ; q)_{\infty}}\right) \\
=\sum_{0 \leq y_{1}, \ldots, y_{m} \leq n-1} q^{y_{2}+2 y_{3}+\cdots+(m-1) y_{m}} a^{y_{1}+\cdots+y_{m}} E\left(\frac{\xi^{y_{1}+\cdots+y_{m}}}{\left(a q^{m} \xi ; q\right)_{\infty}}\right) \\
=\frac{1}{\left(a q^{m}, a x q^{m} ; q\right)_{\infty}} \\
\quad \times \sum_{0 \leq y_{1}, \ldots, y_{m} \leq n-1} q^{y_{2}+2 y_{3}+\cdots+(m-1) y_{m}} a^{y_{1}+\cdots+y_{m}} \varphi_{y_{1}+\cdots+y_{m}}^{\left(a q^{m}\right)}(x \mid q) .
\end{gathered}
$$

Hence, we get the left hand side of (5-2):

$$
\begin{aligned}
& \lim _{n \rightarrow \infty} E\left(\frac{\left[1-(a \xi)^{n}\right]\left[1-(a q \xi)^{n}\right] \cdots\left[1-\left(a q^{m-1} \xi\right)^{n}\right]}{(a \xi ; q)_{\infty}}\right) \\
&= \frac{1}{\left(a q^{m}, a x q^{m} ; q\right)_{\infty}} \\
& \quad \times \sum_{y_{1}, \ldots, y_{m} \geq 0} q^{y_{2}+2 y_{3}+\cdots+(m-1) y_{m}} a^{y_{1}+\cdots+y_{m}} \varphi_{y_{1}+\cdots+y_{m}}^{\left(a q^{m}\right)}(x \mid q) .
\end{aligned}
$$

Substituting (5-3) and (5-4) into (5-2) gives

$$
\sum_{y_{1}, \ldots, y_{m} \geq 0} q^{y_{2}+2 y_{3}+\cdots+(m-1) y_{m}} a^{y_{1}+\cdots+y_{m}} \varphi_{y_{1}+\cdots+y_{m}}^{\left(a q^{m}\right)}(x \mid q)=\frac{1}{(a, a x ; q)_{m}} .
$$

Using Theorem 3.1 with $a=q^{m}$ and $x=a x$ gives

$$
\sum_{k=0}^{\infty}\left[\begin{array}{c}
m+k-1 \\
k
\end{array}\right] a^{k} x^{k}=\frac{1}{(a x ; q)_{m}} .
$$

Substituting (5-6) into (5-5) and comparing the coefficients of $x^{n}$ gives (5-1).

\section{An extension of the Rogers-Ramanujan identities}

The well-known Rogers-Ramanujan identities are

$$
\begin{aligned}
& \sum_{m=0}^{\infty} \frac{q^{m^{2}}}{(q ; q)_{m}}=\frac{1}{\left(q, q^{4} ; q^{5}\right)_{\infty}}, \\
& \sum_{m=0}^{\infty} \frac{q^{m^{2}+m}}{(q ; q)_{m}}=\frac{1}{\left(q^{2}, q^{3} ; q^{5}\right)_{\infty}} .
\end{aligned}
$$

There are many proofs of this beautiful pair of identities. Baxter's [1982] is based on the statistical mechanics, and the proof of Lepowsky and Milne [1978] 
uses the character formula on an infinite dimensional Lie algebra. We use our probability distribution to derive an extension of the Rogers-Ramanujan identities.

Theorem 6.1. We have

$$
\begin{aligned}
& \sum_{m=n}^{\infty} \frac{q^{m^{2}}}{(q ; q)_{m-n}}=\frac{1}{(q ; q)_{\infty}} \\
& \times\left(q^{n}+\sum_{k=1}^{\infty} \sum_{l=0}^{n}\left[\begin{array}{c}
n \\
l
\end{array}\right] \frac{(-1)^{k}\left(q^{k} ; q\right)_{l}(q ; q)_{2 k}}{\left(1-q^{k}\right)(q ; q)_{2 k+1-l}} q^{5\left(\begin{array}{c}
k \\
2
\end{array}\right)+k(n+2-l)}\left(1-q^{2 k}+q^{4 k+1}\right)\right. \\
& \left.\quad-\sum_{k=1}^{\infty} \sum_{l=0}^{n}\left[\begin{array}{c}
n \\
l
\end{array}\right] \frac{(-1)^{k}\left(q^{k} ; q\right)_{l}(q ; q)_{2 k}}{\left(1-q^{k}\right)(q ; q)_{2 k+1-l}} q^{5\left(\begin{array}{c}
k \\
2
\end{array}\right)+k(n+4-l)+1-l}\right) .
\end{aligned}
$$

Proof. By Watson's $q$-Whipple transformation formula [Watson 1929],

$$
\begin{aligned}
{ }_{8} \phi_{7}\left(\begin{array}{c}
a, q \sqrt{a},-q \sqrt{a}, b, c, d, e, q^{-n} \\
\sqrt{a},-\sqrt{a}, q a / b, q a / c, q a / d, q a / e, q^{n+1} a
\end{array} ; q, \frac{q^{2+n} a^{2}}{b c d e}\right) \\
=\frac{(q a, q a / b c ; q)_{n}}{(q a / b, q a / c)_{n}}{ }_{4} \phi_{3}\left(\begin{array}{c}
q^{-n}, b, c, q a / d e \\
q a / d, q a / e, q^{-n} b c / a
\end{array} ; q, q\right) .
\end{aligned}
$$

Letting $b, c, d, e, n \rightarrow \infty$ in this equation gives

$$
\begin{aligned}
\sum_{m=0}^{\infty} \frac{q^{m^{2}} a^{m}}{(q ; q)_{m}}=\frac{1}{(a q ; q)_{\infty}}+\sum_{k=1}^{\infty} \frac{(-1)^{k} q^{5\left(\begin{array}{c}
k \\
2
\end{array}\right)+2 k}}{(q ; q)_{k}} \cdot \frac{a^{2 k}}{\left(a q^{k} ; q\right)_{\infty}} & \\
& -\sum_{k=1}^{\infty} \frac{(-1)^{k} q^{5\left(\begin{array}{c}
k \\
2
\end{array}\right)+4 k}}{(q ; q)_{k}} \cdot \frac{a^{2 k+1}}{\left(a q^{k} ; q\right)_{\infty}} \quad \text { for }|a| \leq 1 .
\end{aligned}
$$

Then letting $a=\xi$ gives

$$
\begin{aligned}
\sum_{m=0}^{\infty} \frac{q^{m^{2}} \xi^{m}}{(q ; q)_{m}}=\frac{1}{(\xi q ; q)_{\infty}}+\sum_{k=1}^{\infty} \frac{(-1)^{k} q^{5\left(\begin{array}{l}
k \\
2
\end{array}\right)+2 k}}{(q ; q)_{k}} & \cdot \frac{\xi^{2 k}}{\left(\xi q^{k} ; q\right)_{\infty}} \\
& -\sum_{k=1}^{\infty} \frac{(-1)^{k} q^{5\left(\begin{array}{l}
k \\
2
\end{array}\right)+4 k}}{(q ; q)_{k}} \cdot \frac{\xi^{2 k+1}}{\left(\xi q^{k} ; q\right)_{\infty}} .
\end{aligned}
$$

where $\xi$ is a random variable with distribution $W(x ; q)$ and $-1<x<0$. Applying the expectation operator $E$ to both sides of the above, we get

$$
\begin{aligned}
E\left(\sum_{m=0}^{\infty} \frac{q^{m^{2}} \xi^{m}}{(q ; q)_{m}}\right)=E\left(\frac{1}{(\xi q ; q)_{\infty}}\right) & +E\left(\sum_{k=1}^{\infty} \frac{(-1)^{k} q^{5\left(\begin{array}{c}
k \\
2
\end{array}\right)+2 k}}{(q ; q)_{k}} \cdot \frac{\xi^{2 k}}{\left(\xi q^{k} ; q\right)_{\infty}}\right) \\
& -E\left(\sum_{k=1}^{\infty} \frac{(-1)^{k} q^{5\left(\begin{array}{c}
k \\
2
\end{array}\right)+4 k}}{(q ; q)_{k}} \cdot \frac{\xi^{2 k+1}}{\left(\xi q^{k} ; q\right)_{\infty}}\right)
\end{aligned}
$$


Since $\left|q^{m^{2}} \xi^{m} /(q ; q)_{m}\right| \leq q^{m^{2}} /(q ; q)_{m}$ and the series $\sum_{m=0}^{\infty} q^{m^{2}} /(q ; q)_{m}$ converges absolutely, using Lebesgue's dominated convergence theorem and (2-6) gives the left hand side of (6-3):

$$
E\left(\sum_{m=0}^{\infty} \frac{q^{m^{2}} \xi^{m}}{(q ; q)_{m}}\right)=\sum_{m=0}^{\infty} \frac{q^{m^{2}} E\left\{\xi^{m}\right\}}{(q ; q)_{m}}=\sum_{m=0}^{\infty} \frac{q^{m^{2}} h_{m}(x \mid q)}{(q ; q)_{m}} .
$$

On the other hand, using (2-6) gives

$$
\begin{aligned}
E\left(\frac{1}{(\xi q ; q)_{\infty}}\right) & =\frac{1}{(q, q x ; q)_{\infty}}, \\
E\left(\frac{\xi^{2 k}}{\left(\xi q^{k} ; q\right)_{\infty}}\right) & =\frac{1}{\left(q^{k}, q^{k} x ; q\right)_{\infty}} \varphi_{2 k}^{\left(q^{k}\right)}(x \mid q), \\
E\left(\frac{\xi^{2 k+1}}{\left(\xi q^{k} ; q\right)_{\infty}}\right) & =\frac{1}{\left(q^{k}, q^{k} x ; q\right)_{\infty}} \varphi_{2 k+1}^{\left(q^{k}\right)}(x \mid q) .
\end{aligned}
$$

It is easy to see that

$$
\left|\frac{(-1)^{k} q^{5\left(\begin{array}{c}
k \\
2
\end{array}\right)+4 k}}{(q ; q)_{k}} \cdot \frac{\xi^{2 k+1}}{\left(\xi q^{k} ; q\right)_{\infty}}\right| \leq\left|\frac{(-1)^{k} q^{5\left(\begin{array}{c}
k \\
2
\end{array}\right)+2 k}}{(q ; q)_{k}} \cdot \frac{\xi^{2 k}}{\left(\xi q^{k} ; q\right)_{\infty}}\right| \leq \frac{q^{5\left(\begin{array}{l}
k \\
2
\end{array}\right)+2 k}}{(q ; q)_{k}(q ; q)_{\infty}}
$$

and the series $\sum_{k=0}^{\infty} q^{5\left(\begin{array}{c}k \\ 2\end{array}\right)+2 k} /\left((q ; q)_{k}(q ; q)_{\infty}\right)$ is converges absolutely. Using Lebesgue's dominated convergence theorem and (6-5), (6-6) and (6-7) gives the right hand side of (6-3):

$$
\begin{aligned}
& E\left(\frac{1}{(\xi q ; q)_{\infty}}\right)+E\left(\sum_{k=1}^{\infty} \frac{(-1)^{k} q^{5\left(\begin{array}{c}
k \\
2
\end{array}\right)+2 k}}{(q ; q)_{k}} \cdot \frac{\xi^{2 k}}{\left(\xi q^{k} ; q\right)_{\infty}}\right) \\
& \quad-E\left(\sum_{k=1}^{\infty} \frac{(-1)^{k} q^{5\left(\begin{array}{c}
k \\
2
\end{array}\right)+4 k}}{(q ; q)_{k}} \cdot \frac{\xi^{2 k+1}}{\left(\xi q^{k} ; q\right)_{\infty}}\right) \\
& =E\left(\frac{1}{(\xi q ; q)_{\infty}}\right)+\sum_{k=1}^{\infty} \frac{(-1)^{k} q^{5\left(\begin{array}{c}
k \\
2
\end{array}\right)+2 k}}{(q ; q)_{k}} E\left(\frac{\xi^{2 k}}{\left(\xi q^{k} ; q\right)_{\infty}}\right) \\
& =\frac{1}{(q, q x ; q)_{\infty}}+\sum_{k=1}^{\infty} \frac{(-1)^{k} q^{5\left(\begin{array}{l}
k \\
2
\end{array}\right)+2 k}}{(q ; q)_{k}} \frac{1}{\left(q^{k}, q^{k} x ; q\right)_{\infty}} \frac{(-1)^{k} q^{5\left(\begin{array}{l}
k \\
2
\end{array}\right)+4 k}}{(q ; q)_{k}} E\left(\frac{\xi^{2 k+1}}{\left(\xi q^{k} ; q\right)_{\infty}}\right) \\
& -\sum_{k=1}^{\infty} \frac{(-1)^{k} q^{5\left(\begin{array}{c}
k \\
2
\end{array}\right)+4 k}}{(q ; q)_{k}} \frac{1}{\left(q^{k}, q^{k} x ; q\right)_{\infty}} \varphi_{2 k+1}^{\left(q^{k}\right)}(x \mid q)
\end{aligned}
$$




$$
\begin{aligned}
& =\frac{1}{(q, q x ; q)_{\infty}} \\
& \quad+\frac{1}{(q, x ; q)_{\infty}} \sum_{k=1}^{\infty} \frac{(-1)^{k}(x ; q)_{k}}{1-q^{k}} q^{5\left(\begin{array}{l}
k \\
2
\end{array}\right)+2 k}\left(\varphi_{2 k}^{\left(q^{k}\right)}(x \mid q)-q^{2 k} \varphi_{2 k+1}^{\left(q^{k}\right)}(x \mid q)\right) .
\end{aligned}
$$

Substituting this and (6-4) into (6-3) gives

$$
\begin{aligned}
& \sum_{m=0}^{\infty} \frac{q^{m^{2}} h_{m}(x \mid q)}{(q ; q)_{m}}=\frac{1}{(q, q x ; q)_{\infty}} \\
& \quad+\frac{1}{(q, x ; q)_{\infty}} \sum_{k=1}^{\infty} \frac{(-1)^{k}(x ; q)_{k}}{1-q^{k}} q^{5\left(\begin{array}{l}
k \\
2
\end{array}\right)+2 k}\left(\varphi_{2 k}^{\left(q^{k}\right)}(x \mid q)-q^{2 k} \varphi_{2 k+1}^{\left(q^{k}\right)}(x \mid q)\right),
\end{aligned}
$$

where $-1<x<0$. By analytic continuation, we may replace the assumption $-1<x<0$ by $|x|<1$.

Substituting the expansion

$$
\frac{1}{(z ; q)_{\infty}}=\sum_{l=0}^{\infty} \frac{z^{l}}{(q ; q)_{l}}
$$

into the last, we have

$$
\begin{aligned}
& \sum_{m=0}^{\infty} \frac{q^{m^{2}} h_{m}(x \mid q)}{(q ; q)_{m}}=\frac{1}{(q ; q)_{\infty}} \sum_{l=0}^{\infty} \frac{q^{l} x^{l}}{(q ; q)_{l}} \\
& \quad+\frac{1}{(q ; q)_{\infty}} \sum_{k=1}^{\infty} \sum_{l=0}^{\infty}\left(\frac{q^{k l} x^{l}}{(q ; q)_{l}} \cdot \frac{(-1)^{k} q^{5\left(\begin{array}{l}
k \\
2
\end{array}\right)+2 k}}{1-q^{k}}\left(\varphi_{2 k}^{\left(q^{k}\right)}(x \mid q)-q^{2 k} \varphi_{2 k+1}^{\left(q^{k}\right)}(x \mid q)\right)\right) .
\end{aligned}
$$

Comparing the coefficients of $x^{n}$ in this identity gives

$$
\begin{aligned}
& \sum_{m=n}^{\infty} \frac{q^{m^{2}}\left[\begin{array}{l}
m \\
n
\end{array}\right]}{(q ; q)_{m}}=\frac{q^{n}}{(q ; q)_{\infty}(q ; q)_{n}} \\
& \quad+\frac{1}{(q ; q)_{\infty}} \sum_{k=1}^{\infty} \sum_{l=0}^{n} \frac{(-1)^{k}\left(q^{k} ; q\right)_{l}}{\left(1-q^{k}\right)(q ; q)_{n-l}} q^{5\left(\begin{array}{l}
k \\
2
\end{array}\right)+k(n+2-l)}\left(\left[\begin{array}{c}
2 k \\
l
\end{array}\right]+q^{2 k}\left[\begin{array}{c}
2 k+1 \\
l
\end{array}\right]\right),
\end{aligned}
$$

which can be written as Theorem 6.1 .

The Rogers-Ramanujan identities are special cases of Theorem 6.1. Letting $n=0$ and then applying the Jacobi triple product identity [Andrews et al. 1999]

$$
\sum_{n=-\infty}^{\infty}(-1)^{n} q^{\left(\begin{array}{c}
n \\
2
\end{array}\right)} x^{n}=(q, x, q / x ; q)_{\infty}
$$


leads to the Rogers-Ramanujan identity (6-1). In fact, when $n=0$, we have

$$
\begin{aligned}
\sum_{m=0}^{\infty} \frac{q^{m^{2}}}{(q ; q)_{m}} & =\frac{1}{(q ; q)_{\infty}}\left(1+\sum_{k=1}^{\infty}(-1)^{k}\left(1+q^{k}\right) q^{5\left(\begin{array}{l}
k \\
2
\end{array}\right)+2 k}\right) \\
& =\frac{1}{(q ; q)_{\infty}} \sum_{k=-\infty}^{\infty}(-1)^{k} q^{5\left(\begin{array}{l}
k \\
2
\end{array}\right)+2 k} \\
& =\frac{\left(q^{5}, q^{2}, q^{3} ; q^{5}\right)_{\infty}}{(q ; q)_{\infty}}=\frac{1}{\left(q, q^{4} ; q^{5}\right)_{\infty}} .
\end{aligned}
$$

Similarly, the case $n=1$ of Theorem 6.1 results in another identity due to Rogers and Ramanujan:

$$
\begin{aligned}
\sum_{m=0}^{\infty} \frac{q^{m^{2}+m}}{(q ; q)_{m}} & =\sum_{m=0}^{\infty} \frac{q^{m^{2}}}{(q ; q)_{m}}-\sum_{m=1}^{\infty} \frac{q^{m^{2}}}{(q ; q)_{m-1}} \\
& =\frac{1}{(q ; q)_{\infty}}\left(1+\sum_{k=1}^{\infty}\left(1-q^{2 k+1}\right) q^{5\left(\begin{array}{c}
k \\
2
\end{array}\right)+4 k}\right) \\
& =\frac{1}{(q ; q)_{\infty}} \sum_{k=-\infty}^{\infty}(-1)^{k} q^{5\left(\begin{array}{c}
k \\
2
\end{array}\right)+4 k} \\
& =\frac{\left(q, q^{4}, q^{5} ; q^{5}\right)_{\infty}}{(q ; q)_{\infty}}=\frac{1}{\left(q^{2}, q^{3} ; q^{5}\right)_{\infty}} .
\end{aligned}
$$

\section{References}

[Andrews and Askey 1981] G. E. Andrews and R. Askey, "Another $q$-extension of the beta function”, Proc. Amer. Math. Soc. 81:1 (1981), 97-100. MR 81j:33001 Zbl 0471.33001

[Andrews et al. 1999] G. E. Andrews, R. Askey, and R. Roy, Special functions, Encyclopedia of Mathematics and its Applications 71, Cambridge University Press, 1999. MR 2000g:33001 Zbl 0920.33001

[Baxter 1982] R. J. Baxter, Exactly solved models in statistical mechanics, Academic Press, London, 1982. MR 86i:82002a Zbl 0538.60093

[Chapman 2005] R. Chapman, "A probabilistic proof of the Andrews-Gordon identities", Discrete Math. 290:1 (2005), 79-84. MR 2005i:11145 Zbl 1081.11066

[Evans 2002] S. N. Evans, "Elementary divisors and determinants of random matrices over a local field”, Stochastic Process. Appl. 102:1 (2002), 89-102. MR 2004c:15041 Zbl 1075.15500

[Fulman 2001] J. Fulman, "A probabilistic proof of the Rogers-Ramanujan identities", Bull. London Math. Soc. 33:4 (2001), 397-407. MR 2002b:11146 Zbl 1040.11074

[Gasper and Rahman 1990] G. Gasper and M. Rahman, Basic hypergeometric series, Encyclopedia of Mathematics and its Applications 35, Cambridge University Press, 1990. MR 91d:33034 Zbl 0695.33001

[Jackson 1910] F. H. Jackson, “On q-definite integrals”, Quart. J. Pure and Appl. Math 41 (1910), 101-112. JFM 41.0317.04 
[Lepowsky and Milne 1978] J. Lepowsky and S. Milne, "Lie algebraic approaches to classical partition identities", Adv. in Math. 29:1 (1978), 15-59. MR 82f:17005 Zbl 0384.10008

[Liu 2003] Z.-G. Liu, "Some operator identities and $q$-series transformation formulas", Discrete Math. 265:1-3 (2003), 119-139. MR 2004c:33034 Zbl 1021.05010

[Milne 1997] S. C. Milne, "Balanced ${ }_{3} \phi_{2}$ summation theorems for $\mathrm{U}(n)$ basic hypergeometric series”, Adv. Math. 131:1 (1997), 93-187. MR 99d:33025 Zbl 0886.33014

[Rawlings 1997] D. Rawlings, "Absorption processes: models for $q$-identities", Adv. in Appl. Math. 18:2 (1997), 133-148. MR 98b:05010 Zbl 0867.05003

[Srivastava and Jain 1989] H. M. Srivastava and V. K. Jain, "Some multilinear generating functions for $q$-Hermite polynomials", J. Math. Anal. Appl. 144:1 (1989), 147-157. MR 91g:33024a Zbl 0665.33008

[Tannery 1904] J. Tannery, Introduction à la théorie des fonctions d'une variable, I: Nombres irrationnels, ensembles, limites, fonctions élémentaires, dérivées, 2nd ed., A. Hermann, Paris, 1904. JFM 35.0374.01

[Wang 2008] M. Wang, "A remark on Andrews-Askey integral”, J. Math. Anal. Appl. 341:2 (2008), 1487-1494. MR 2009e:33056 Zbl 1142.33006

[Wang 2009a] M. Wang, "Generalizations of Milne's U $(n+1) q$-binomial theorems", Comput. Math. Appl. 58:1 (2009), 80-87. MR 2010f:33027

[Wang 2009b] M. Wang, " $q$-integral representation of the Al-Salam-Carlitz polynomials", Appl. Math. Lett. 22:6 (2009), 943-945. MR 2523611 Zbl 1173.33305

[Wang 2010a] M. Wang, "An extension of the $q$-beta integral with applications", J. Math. Anal. Appl. 365:2 (2010), 653-658. MR 2587068 Zbl 05676137

[Wang 2010b] M. Wang, "A recurring q-integral formula", Appl. Math. Lett. 23:3 (2010), 256-260. MR 2565186 Zbl 1183.33036

[Watson 1929] G. N. Watson, "A new proof of the Rogers-Ramanujan identities", J. London Math. Soc. 4 (1929), 4-9. JFM 55.0219.09

[Zhang 2006] Z. Zhang, "Operator identities and several $U(n+1)$ generalizations of the KalninsMiller transformations”, J. Math. Anal. Appl. 324:2 (2006), 1152-1167. MR 2008b:33043 Zbl 1113.33020

[Zhang and Liu 2006] Z. Zhang and M. Liu, "Applications of operator identities to the multiple $q$ binomial theorem and $q$-Gauss summation theorem", Discrete Math. 306:13 (2006), 1424-1437. MR 2007f:33025 Zbl 1095.05002

Received February 21, 2009. Revised December 12, 2009.

MingJIN WANG

Department of Applied Mathematics

CHANGZHOU UNIVERSITY

CHANGZHOU 213164

CHINA

wmj@cczu.edu.cn 


\title{
PACIFIC JOURNAL OF MATHEMATICS
}

\author{
http://www.pjmath.org \\ Founded in 1951 by \\ E. F. Beckenbach (1906-1982) and F. Wolf (1904-1989)
}

\section{EDITORS}

V. S. Varadarajan (Managing Editor)

Department of Mathematics

University of California

Los Angeles, CA 90095-1555

pacific@math.ucla.edu

Vyjayanthi Chari

Department of Mathematics

University of California

Riverside, CA 92521-0135

chari@math.ucr.edu

Robert Finn

Department of Mathematics Stanford University

Stanford, CA 94305-2125

finn@math.stanford.edu

Kefeng Liu

Department of Mathematics

University of California

Los Angeles, CA 90095-1555

liu@math.ucla.edu
Darren Long

Department of Mathematics

University of California

Santa Barbara, CA 93106-3080

long@math.ucsb.edu

Jiang-Hua Lu

Department of Mathematics

The University of Hong Kong

Pokfulam Rd., Hong Kong jhlu@maths.hku.hk

Alexander Merkurjev

Department of Mathematics

University of California

Los Angeles, CA 90095-1555

merkurev@math.ucla.edu
Sorin Popa

Department of Mathematics University of California

Los Angeles, CA 90095-1555 popa@math.ucla.edu

Jie Qing

Department of Mathematics

University of California

Santa Cruz, CA 95064

qing@cats.ucsc.edu

Jonathan Rogawski

Department of Mathematics

University of California

Los Angeles, CA 90095-1555

jonr@math.ucla.edu

\section{PRODUCTION}

pacific@math.berkeley.edu

\begin{abstract}
Silvio Levy, Scientific Editor Matthew Cargo, Senior Production Editor
\end{abstract}
ACADEMIA SINICA, TAIPEI

CALIFORNIA INST. OF TECHNOLOGY

INST. DE MATEMÁTICA PURA E APLICADA

KEIO UNIVERSITY

MATH. SCIENCES RESEARCH INSTITUTE

NEW MEXICO STATE UNIV.

OREGON STATE UNIV.

\section{SUPPORTING INSTITUTIONS}

STANFORD UNIVERSITY
UNIV. OF BRITISH COLUMBIA
UNIV. OF CALIFORNIA, BERKELEY
UNIV. OF CALIFORNIA, DAVIS
UNIV. OF CALIFORNIA, LOS ANGELES
UNIV. OF CALIFORNIA, RIVERSIDE
UNIV. OF CALIFORNIA, SAN DIEGO
UNIV. OF CALIF., SANTA BARBARA

UNIV. OF CALIF., SANTA CRUZ

UNIV. OF MONTANA

UNIV. OF OREGON

UNIV. OF SOUTHERN CALIFORNIA

UNIV. OF UTAH

UNIV. OF WASHINGTON

WASHINGTON STATE UNIVERSITY

These supporting institutions contribute to the cost of publication of this Journal, but they are not owners or publishers and have no responsibility for its contents or policies.

See inside back cover or www.pjmath.org for submission instructions.

The subscription price for 2010 is US \$420/year for the electronic version, and \$485/year for print and electronic.

Subscriptions, requests for back issues from the last three years and changes of subscribers address should be sent to Pacific Journal of Mathematics, P.O. Box 4163, Berkeley, CA 94704-0163, U.S.A. Prior back issues are obtainable from Periodicals Service Company, 11 Main Street, Germantown, NY 12526-5635. The Pacific Journal of Mathematics is indexed by Mathematical Reviews, Zentralblatt MATH, PASCAL CNRS Index, Referativnyi Zhurnal, Current Mathematical Publications and the Science Citation Index.

The Pacific Journal of Mathematics (ISSN 0030-8730) at the University of California, c/o Department of Mathematics, 969 Evans Hall, Berkeley, CA 94720-3840, is published monthly except July and August. Periodical rate postage paid at Berkeley, CA 94704, and additional mailing offices. POSTMASTER: send address changes to Pacific Journal of Mathematics, P.O. Box 4163, Berkeley, CA 94704-0163.

PJM peer review and production are managed by EditFLOW ${ }^{\mathrm{TM}}$ from Mathematical Sciences Publishers.

PUBLISHED BY PACIFIC JOURNAL OF MATHEMATICS

at the University of California, Berkeley 94720-3840

A NON-PROFIT CORPORATION

Typeset in LATEX

Copyright $(\mathrm{C} 2010$ by Pacific Journal of Mathematics 


\section{PACIFIC JOURNAL OF MATHEMATICS}

Volume $247 \quad$ No. $1 \quad$ September 2010

Classification results for easy quantum groups

TEOdor BANiCA, StePhen CURRAN and Roland SPEICHER

Batalin-Vilkovisky coalgebra of string topology

XiAOJUn CHEN and WeE LiAnG GAN

Invariant Finsler metrics on polar homogeneous spaces

SHAOQIANG DENG

A proof of the Concus-Finn conjecture

KIRK E. LANCASTER

The existence and monotonicity of a three-dimensional transonic shock in a finite nozzle with axisymmetric exit pressure

JUn Li, ZHOUPING XIN and HUICHENG YiN

Bi-Hamiltonian flows and their realizations as curves in real semisimple homogeneous manifolds

GLORIA MARÍ BEFFA

Closed orbits of a charge in a weakly exact magnetic field

WILL J. MERRY

Ringel-Hall algebras and two-parameter quantized enveloping algebras

XIN TANG

A new probability distribution with applications

MINGJIN WANG 